\title{
An application of combinatorial techniques to a topological problem
}

\section{Ludvik Janos}

The following statement is proved: Let $X$ be a set having at most continuously many elements and $f: X \rightarrow X$ a mapping such that each iteration $f^{n} \quad(n=1,2, \ldots)$ has a unique fixed point. Then for every number $c \in(0,1)$ there exists a metric $\rho$ on $X$ such that the metric space $(X, \rho)$ is separable and the mapping $f$ is a contraction with the Lipschitz constant $c$.

\section{Introduction}

In recent two decades different mathematicians asked the following question: Given an abstract set $X$ and a mapping $f: X \rightarrow X$, does there exist a non-trivial topology on $X$ which would render $f$ continuous and would satisfy at the same time some prescribed conditions (compactness, separability, metrizability, Hausdorff property, and so forth)? de Groot and de Vries [3] proved that if $X$ has at most continuously many elements then for every $f: X \rightarrow X$ there exists a non-discrete separable metric topology on $X$ rendering $f$ continuous. Bessaga [2] obtained the following result (a converse to the Banach fixed point theorem).

THEOREM 1 (Bessaga). Let $X$ be a set and $f: X \rightarrow X$ such that all the iterates $f^{n}$ have a unique fixed point. Assuming the weak (countable) form of the axiom of choice, then for any $c \in(0,1)$ there exists a complete metric on $X$ rendering $f$ a c-contraction.

The purpose of this note is to show that in case $X$ has at most continuously many elements then the separability of the metric in the above Received 9 July 1973. 
theorem can be claimed. In the construction of this metric we will use the following combinatorial theorem of Ramsey (see, for example, [1]).

THEOREM 2 (Ramsey). If the set of all zonordered pairs $\{n, m\}$ of natural numbers $N$ is decomposed in finite number of sets, say $R_{1}, R_{2}, \ldots, R_{k}$, that is,

$$
\{A|| A \mid=2 \text { and } A \subset N\}=R_{1} \cup R_{2} \cup \ldots \cup R_{k}
$$

then there exists an infinite subset $M \subset N$ and an index $i \in\{1,2, \ldots, k\}$ such that alz pairs $\{n, m\} \subset M$ belong to $R_{i}$.

Finally we will need the following result of Meyers [4].

THEOREM 3 (Meyers). If $X$ is a metrizable topological space and $f: X \rightarrow X$ a continuous mopping satisfying:

(i) $f$ has a unique fixed point $a$, that is, $f(a)=a$;

(ii) for every $x \in X$ the sequence of iterates $x, f(x), f^{2}(x), \ldots$ converges to $a$;

(iii) there exists a neighbourhood $U_{a}$ of a such that for any neighbourhood $V_{a}$ of a there exists $n_{0}$ such that $n \geq n_{0}$ implies $f^{n}\left(u_{a}\right) \subset v_{a}$;

then for every $c \in(0,1)$ there exists a metric on $X$ which is compatible with the topology of $x$ and with respect to which $f$ is a c-contraction.

\section{Proof of the theorem}

Let $X$ be an abstract set with at most continuously many elements and let $f: X \rightarrow X$ satisfy the conditions of Theorem 1 . Choosing $c=\frac{1}{2}$ we denote by $\rho$ the corresponding metric on $X$ existing by this theorem. If $a$ is the fixed point of $f$ we define the sets $A_{n}$ ( $n$ integer) by:

$$
A_{n}=\left\{x \mid x \in X \text { and } 2^{n-1}<\rho(a, x) \leq 2^{n}\right\} \text {. }
$$


Thus we obtain a disjoint partition of $X$ in the form $X=\{a\} \cup \stackrel{+\infty}{\cup} A_{n}$ satisfying the condition that the image $f\left(A_{n}\right)$ of $A_{n}$ under $f^{\prime}$ is contained in $\{a\} \cup \underset{-\infty}{n-1} A_{k}$. Once this result is achieved, we disregard the metric $\rho$ (since it is not separable in ge:eral) and proceed in the following way.

We consider the subset $\{0\} \cup \underset{-\infty}{\bigcup_{-\infty}^{+\infty}} C_{n}$ of the euclidean plane where 0 is the origin and $C_{n}$ is the circle with centre in 0 and of radius $2^{n}$. Since each set $A_{n}$ has at most continuously many elements one can identify $A_{n}$ with a certain subset $B_{n} \subset C_{n}$ of $C_{n}$. Doing this for every $n$ and identifying $a$ with the origin 0 , our set $X$ can be thought of as the set $\{0\} \cup \bigcup_{-\infty}^{+\infty} B_{n}$. Denoting by $d_{2}$ the euclidean metric we thus obtain a separable metric space $\left(x, d_{2}\right)$ and it follows from the definition that each subset $\{0\} \cup \bigcup_{-\infty}^{n} B_{k}$ is totally bounded and invariant under $f$.

We now define a new metric $d_{2}^{*}$ on $X$ with respect to which $f$ will be continuous as follows:

$$
d_{2}^{*}(x, y)=\sup _{n \geq 0} d_{2}\left(f^{n}(x), f^{n}(y)\right)
$$

for $x, y \in X$ and where $f^{0}(x)$ stands for $x$. It is clear that $d_{2}^{*}$ is a metric and that $f$ is continuous with respect to $d_{2}^{*}$, since from the definition it follows immediately that $f$ is non-expanding:

$$
d_{2}^{*}(f(x), f(y)) \leq d_{2}^{*}(x, y)
$$

Since the circles $C_{n}$ shrink to 0 it follows that for each pair $x, y \in X$ there is a number $n=n(x, y)$ such that 
$d_{2}^{*}(x, y)=d_{2}\left(f^{n}(x), f^{n}(y)\right)$. In order to show that the sets $\{0\} \cup U_{-\infty}^{n} B_{k}$ are totally bounded also with respect to the metric $d_{2}^{*}$ we need the following.

LEMMA. Let $(Y, d)$ be a totally bounded metric space and let $f: y+y$ (not necessarily continuous) be such that the diameters $\delta_{n}$ of the iterated images $f^{n}(Y)$ converge to zero as $n \rightarrow \infty$. Then the metric $d^{*}$ on $Y$ defined by

$$
d^{*}(x, y)=\sup _{n \geq 0} d\left(f^{n}(x), f^{n}(y)\right)
$$

is also totally bounded.

Proof. First we observe that due to $\delta_{n} \rightarrow 0$ there is an integer $n=n(x, y)$ for each pair of points $x, y \in Y$ such that $d^{*}(x, y)=d\left(f^{n}(x), f^{n}(y)\right)$. Now if $d^{*}$ were not totally bounded there would be a number $\varepsilon>0$ and a sequence $\left\{x_{k}\right\} \subset y$ such that

$$
d^{*}\left(x_{k}, x_{2}\right) \geq \varepsilon \text { for all } k \neq 2 \text {. }
$$

But this would mean that there is a function $n(k, l)$ on the set of all unordered pail $\{k, l\}$ of natural numbers such that $d\left(f^{n(k, l)}\left(x_{k}\right), f^{n(k, l)}\left(x_{\eta}\right)\right) \geq \varepsilon$ for all pairs $\{k, \tau\} \subset N$. Again due to the shrinkage $\delta_{n} \rightarrow 0$ it is obvious that the function $n(k, \tau)$ must be bounded and so its range consists of finite numbers of values, say $n_{1}, n_{2}, \ldots, n_{r}$. But Theorem 2 would then imply that for some $i \in\{1,2, \ldots, r\}$ the inequality $d\left(f^{n}{ }^{n}\left(x_{k}\right), f^{n} i\left(x_{l}\right)\right) \geq \varepsilon$ would hold for some infinite subset of indices which would contradict the assumption that $d$ is totally bounded. This proves that $d^{*}$ must be totally bounded as well.

Observing that the restriction of $f: X \rightarrow X$ to the invariant subset $X_{n}=\{0\} \cup \bigcup_{-\infty}^{n} B_{k}$ satisfies the hypothesis of our lemma we arrive at the 
following conclusion.

As a countable union of totally bounded sets, $\left(X, d_{2}^{*}\right)$ is a separable metric space and $f: X \rightarrow X$ a continuous mapping. Since $d_{2}^{*} \geq d_{2}$ it follows that the topology generated by $d_{2}^{*}$ is in general finer than the Euclizean generated by $d_{2}$. Since each set $x_{n}$ is $d_{2}$-open, it is also $d_{2}^{*}$-open and observing that for each $x \in X$ we have $d_{2}^{*}(0, x)=d_{2}(0, x)$ it follows that each open neighbourhood of 0 with respect to $d_{2}^{*}$ contains some set $x_{n}$. Since $f\left(x_{n}\right) \subset x_{n-1}$ this implies that the conditions of Theorem 3 are satisfied for the topology generated by $d_{2}^{*}$ and our theorem follows from Theorem 3.

REMARK. It is so far not known if the space $\left(x, d_{2}^{*}\right)$ can be assumed topologically complete. In this case the result of Meyers [4] would furnish at the same time a separable and complete metric. So it appears that the gain of separability was paid for by the loss of completeness.

\section{References}

[1] Philip Bacon, "Extending a complete metric", Amer. Math. Monthly 75 (1968), 642-643.

[2] C. Bessaga, "On the converse of the Banach 'fixed-point principle'", Colloq. Math. 7 (1959), 41-43.

[3] J. de Groot and H. de Vries, "Metrization of a set which is mapped into itself", Quart. J. Math. Oxford (2) 9 (1958), 144-148.

[4] Philip R. Meyers, "A converse to Banach's contraction theorem", $J$. Res. Nat. Bur. Standards Sect. B 71 (1967), 73-76.

Department of Mathematics, University of Newcastle, Newcastle, New South wales. 